\title{
Semantic processing in visual word recognition: Activation blocking and domain specificity
}

\author{
MATTHEW S. BROWN, MARTHA ANNE ROBERTS, and DEREK BESNER \\ University of Waterloo, Waterloo, Ontario, Canada
}

\begin{abstract}
Lexical decision to a target is typically facilitated when a related prime is simply read, but is typically eliminated if subjects carry out a letter search on the prime when probe and prime appear simultaneously. Three experiments involving the addition of a task-irrelevant word to this prime search paradigm address (1) Stolz and Besner's $(1996,1998)$ account in which semantic priming is eliminated because letter search on the prime instantiates an activation block between lexical and semantic levels of representation, (2) Chiappe, Smith, and Besner's (1996) account that activation blocking is domain specific, and (3) Neely and Kahan's (2001) claim that semantic activation is context independent and capacity free. The results are (1) consistent with the account that activation blocking is general to a level, rather than item specific, (2) consistent with the account that activation blocking is domain specific, and (3) inconsistent with the claim that semantic activation is context independent and capacity free.
\end{abstract}

In the semantic priming paradigm, a prime stimulus is typically followed by a target that is to be processed in some way (e.g., naming, lexical decision). Target processing is faster when the prime is semantically/associatively related to the target word than when it is not. Priming is even observed when subjects claim not to see the prime because of masking, or when the target is unexpected because of a very low relatedness probability (see review by Neely, 1991; Neely \& Kahan, 2001). However, the context in which the prime is processed is also important in determining its effect on the target. For example, semantic priming in lexical decision and naming can be eliminated ${ }^{1}$ by asking subjects to search the prime word for a letter prior to their responding to the target (e.g., Friedrich, Henik, \& Tzelgov, 1991; Henik, Friedrich, \& Kellogg, 1983; Henik, Friedrich, Tzelgov, \& Tramer, 1994; Smith, Theodor, \& Franklin, 1983; Stolz \& Besner, 1996, 1998).

\section{How Does Semantic Priming Arise?}

Stolz and Besner $(1996,1998)$ discussed both semantic priming and its elimination in the context of McClelland and Rumelhart's (1981; McClelland, 1987) interactive activation (IA) framework. The framework has three levels: a letter level, a lexical level, and a semantic level. There are excitatory feed-forward connections between levels; within-level connections are purely competitive. Activation cascades forward across levels and there is also feedback between adjacent levels.

This research was supported by NSERC Grant AO998 to D.B. We are grateful to two anonymous reviewers for their comments and to John Wixted for editorial assistance. Correspondence should be addressed to D. Besner, Psychology Department, University of Waterloo, Waterloo, ON, N2L 3G1 Canada (e-mail: ms2brown@watarts.uwaterloo.ca, ma3rober@watarts.uwaterloo.ca, or dbesner@watarts.uwaterloo.ca).
As Stolz and Besner (1996) noted, the IA framework explicitly denies the spread of activation within a level. Some theorists have taken this to be evidence that semantic priming cannot be accommodated within the IA framework (e.g., Taft, 1991, pp. 23-24). In contrast, Stolz and Besner (1996) proposed that the broadening of the assumption of between-level activation to include lexical level-to-semantic level activation for related concepts is sufficient to produce semantic priming within the IA framework, provided that within-level competition does not drive the activation of weaker related candidates to baseline. More specifically, the presentation of a prime (e.g., CAT) activates the appropriate letter level representations that then feed activation forward to the lexical level, where the best-fit candidate CAT is activated. Activation cascades forward to semantic level representations of CAT and, less strongly, to that of associates (e.g., DOG; more specifically, semantic features common to DOG and САT). The CAT candidate is the best-fitting representation at the semantic level; however, it is assumed that withinlevel inhibition does not eliminate activation of all competitors. Rather, activation, albeit weakened by withinlevel competition, remains for associates of CAT, such as DOG. Subsequent presentation of the target DOG requires less bottom-up activation for recognition at the semantic level than would be required if DOG had been preceded by an unrelated prime such as DESK (see Stolz, Robidoux, \& Besner, 2000, for simulation work in the context of an IA network that produces semantic priming in this way).

\section{Why Does Letter Search Eliminate Semantic Priming?}

When the prime item and probe letter are presented simultaneously, appropriate letter level representations of the word are activated. Activation feeds forward to the lexical level and the corresponding lexical candidates are 
activated. This activation feeds back to the letter level. Stolz and Besner $(1996,1998)$ assumed that explicit letter identification necessary to complete a letter search on the prime requires more activation at the letter level than do the implicit letter identification processes that take place when the prime is merely read. They also assumed that feedback from the lexical level to the letter level is increased by blocking activation spread from the lexical level to the semantic level. ${ }^{2}$ This activation block eliminates semantic priming following letter search on the prime word. Implementing an activation block is simple from a computational perspective; simply zero out the connection weights between lexical and semantic levels.

Converging evidence that words are processed to the lexical level during letter search on the prime is provided by the observation that morphemic priming is seen in the absence of semantic priming. That is, targets preceded by morphologically related primes (e.g., MARK preceded by MARKED) are processed faster than when preceded by unrelated primes, controlled for orthography and phonology (e.g., MARKET) in an experiment that yielded no semantic priming (Stolz \& Besner, 1998).

\section{The Present Research}

How might one conceptualize the activation block postulated by Stolz and Besner (1996, 1998)? One way to think about it is in terms of mental set, where set is a form of attention. As Henderson (1987) remarks,

Attention-like effects can be produced which greatly change the distribution of activation across the network, and in particular, the role of different levels of representation in the network, simply by biasing the gain between levels, without any structural reorganisation. (p. 190)

What is unclear here is how "level" should be construed. Are all items at the lexical level prevented from sending activation to the semantic level, or is only the prime word being searched prevented from feeding activation to the semantic level? Put another way, is the activation block specific to the prime item being searched or general to all items at the lexical level?

This question is operationalized in Experiments 1 and 2 by adding a second (irrelevant) word to the standard letter search prime display and instructing subjects to ignore it. The experiments will be discussed in greater detail in the Method section. The prime display thus consists of a word and a probe letter (both in red), as well as a second word, in white, that the subjects were instructed to ignore, as is shown in Figure 1.

In Experiment 1, the task-irrelevant event (the ignored prime) was presented either above or below both the word to be searched (the attended prime) and the probe letter string, as is depicted in Figure 1, an equal number of times in a random fashion. If letter search on the prime results in a level specific activation block that prevents all items from sending activation to the semantic level, semantic priming will not be seen for either attended or ignored primes. In contrast, if letter search on the attended prime results in an item specific block, a dissociation should occur, so that the ignored prime produces semantic priming but the attended prime does not.

\section{Is Semantic Activation Automatic?}

It is widely assumed that semantic activation, as indexed by the semantic priming effect, is automatic in the sense that it does not require attentional resources to begin or to run to completion. According to Neely and Kahan (2001), if semantic activation is truly automatic, semantic activation (1) must be fast acting, (2) must be capacity free, (3) can occur without intention, (4) is involuntary or uncontrollable, and (5) can occur without conscious awareness. Although all these characteristics regarding the purported automatic nature of semantic activation warrant investigation, the present experiments focus on the claim that semantic activation is context independent and capacity free. In particular, Neely and Kahan claim that semantic activation for visually presented words should be unaffected by the subject's intention to attend or to ignore that word and by the type of processing required for any number of other elements in the display (i.e., is context independent):

If SA [semantic activation] is strongly automatic, from a foveally presented task-irrelevant word should occur fullblown even when attention should be fully allocated to other task-relevantevents in the display and this should be so re-
Experiment 1

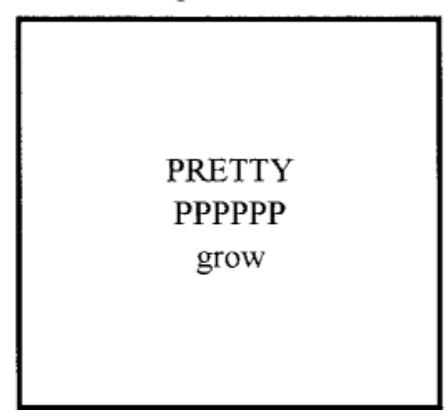

Experiment 2

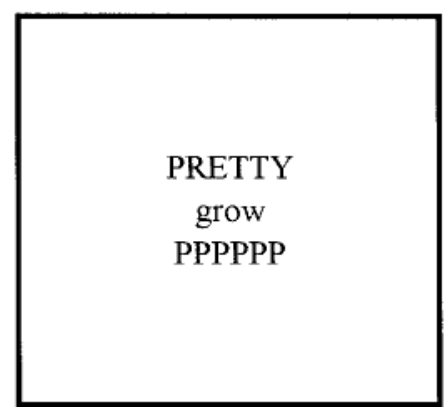

Experiment 3

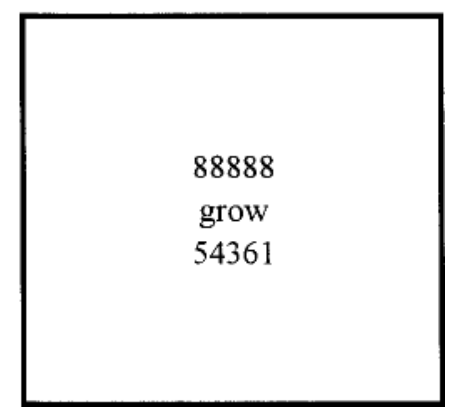

Figure 1. Prime display for Experiments 1, 2, and 3 (characters in lowercase were in white; other characters appeared in red). 
gardless of the type of processing (linguistic or otherwise) that must be performed on those task-relevantevents. (Neely \& Kahan, 2001) p. 73

Given Neely and Kahan's (2001) assertion that semantic activation from a task-irrelevant word occurs full blown, even when attention is fully allocated to other taskrelevant events, the ignored prime in Experiment 1 should produce semantic priming. This did not occur; Experiment 1 yielded no semantic priming for either attended or ignored primes in the letter search condition. In contrast, semantic priming was observed for both attended and ignored primes in the read condition, in which subjects were instructed to read the attended prime and ignore the ignored prime.

Given that the unattended prime was not presented foveally, it might be argued that Experiment 1 is not a strong test of Neely and Kahan's (2001) claim, because they stressed that semantic activation for a task-irrelevant word will occur only if it is presented foveally. Of course, the ignored prime in the read condition must have been processed, since semantic priming was observed. However, it is possible that the focus of attention might have become progressively more narrow as task difficulty increased. Thus, the difficulty of the letter search condition in comparison with the read condition might have resulted in a more narrow focus of attention, with the consequence that the ignored prime might not have been processed in the letter search condition. Experiment 2 addressed this concern by repeating Experiment 1, but presenting the ignored prime at fovea (see Figure 1). Despite this, Experiment 2 failed to produce semantic priming for either attended or ignored primes in the RT data.

\section{Domains of Processing}

Whether or not semantic activation occurs likely depends on a host of factors, but one of the factors that concerns us here is whether the attended and ignored primes belong to the same domain. Chiappe et al. (1996) reported the standard elimination of the semantic priming effect when subjects performed a letter search on the prime. However, semantic priming was observed when subjects were required to perform a color discrimination task on the prime. Chiappe et al. therefore argued that global judgment of color is a domain that does not make demands on the resources that drive the visual word recognition machinery. Hence, there is no need for an activation block, and thus, semantic activation occurs.

Chiappe et al.'s (1996) analysis is extended in Experiment 3 , in which the letter search task was replaced by a digit search task, and the ignored prime word was presented in between the attended prime (now a number string) and the probe digit, as is shown in Figure 1.

Since Arabic numerals and alphabetic print (words) belong to different domains, there is no need to implement an activation block in the visual word recognition system under these conditions. The default set to process to semantics should therefore occur for the ignored prime. Con- sistent with this analysis, Experiment 3 yielded semantic priming.

\section{METHOD}

\section{Subjects}

One hundred twelve University of Waterloo undergraduate students participated (48 in Experiment 1; 32 in Experiment 2; 32 in Experiment 3). All subjects spoke English as their first language. Each subject was paid $\$ 6.00$.

\section{Design}

The letter search condition in Experiment 1 consisted of a 2 (probe type: present vs. absent) $\times 3$ (prime type: attended prime related to target vs. ignored prime related to target vs. attended and ignored primes unrelated to target) $\times 2$ (ignored prime location: above probe string vs. below probe string) $\times 2$ (target type: word vs. nonword) within-subjects design. Target type, probe type, ignored prime location, and prime type conditions were varied randomly throughout the experiment.

\section{Stimulus Materials and List Construction}

A set of 160 associatively and semantically related pairs were selected from several previous studies of priming using lexical decision (Besner \& Smith, 1992; Borowsky \& Besner, 1991; Smith, Besner, \& Miyoshi, 1994). These word pairs were randomly divided into two sets of 80 related word pairs. Half of the subjects received one set of 80 related pairs, and the other half received the other 80 pairs. The 80 related pairs not presented to a particular subject in either the attended or ignored related conditions were randomly recombined to create unrelated pairs. Each related and unrelated word pair was randomly matched with an unrelated word to form a word triplet. A word triplet comprised the three lexical stimuli used on a single trial. One hundred sixty unrelated word pairs were paired with 160 legal nonwords to create the nonword target trials. Counterbalancing was accomplished by rotating the related pairs through both positions in the prime display, as well as through the target display. This was done across subjects in order to ensure that each subject saw each word only once. An additional set of 16 trials served as practice trials and was presented in a separate block prior to the experimental trials.

Each subject received 320 trials consisting of a prime display, followed by a target. Of these trials, half contained a nonword target and half contained a word target. Half of the word targets were unrelated to either the attended prime or the ignored prime. On the other half of the word target trials, the target was related to one of the two prime words. In other words, the attended prime was related to the target on 40 trials (the attended prime condition), and the ignored prime was related to the target on another 40 trials (the ignored prime condition). Thus, the relatedness proportion (the proportion of word targets related to the prime) in both the attended prime and ignored prime conditions was .25 . The two prime words appearing in the same display were never semantically related to one another. All stimuli were 4-6 characters in length and were presented in red (RGB 63,0,0) or white (RGB 63,63,63) on a black background, as dictated by condition. Stimuli were displayed by using the default font in MEL (Schneider, 1988).

\section{Procedure}

The subjects were tested individually and were seated approximately $57 \mathrm{~cm}$ from the computer monitor. General task instructions were displayed on the computer monitor and were also relayed verbally by the experimenter to ensure comprehension. Stimuli were displayed on an ADI Micro Scan color monitor and were controlled by Micro Experimental Laboratory (MEL) software (Schneider, 1988, 1990) running on a Vault PC-compatible computer. Responses were 
executed by a keypress on a computer keyboard. Response latency was measured to the nearest millisecond.

Each trial began with a fixation cross $(+)$, centered on the screen for $200 \mathrm{msec}$. The fixation cross was then replaced by the central presentation of the probe string (e.g., "GGGG" in red). Two primes appeared at the same time as the probe, the attended prime (e.g., "GROW" in red) and the ignored prime (e.g., "pretty" in white), as is shown in Figure 1. The prime display occupied three consecutive text lines, measuring $2.5^{\circ}$ of visual angle vertically, and was centered in the middle of the computer screen. The probe string consisted of the same number of characters as the attended prime. For half of the trials, the attended prime appeared above the probe string, and the ignored prime appeared below it. For the other half of the trials, the positioning of the attended prime and the ignored prime was reversed. The probe character was present in the attended prime for half of the trials and was absent for the other half of the trials. The probe character never appeared in the ignored prime. The subjects were instructed to press the " $z$ " key with their left index finger if the probe character was in the attended prime or to press the "/" key with their right index finger if the probe character was not in the attended prime. The prime display remained on the screen until a response was made. Upon response to the attended prime, the prime display was replaced with the target following a 200-msec interstimulus interval. The target display consisted of a white letter string (in lowercase) that appeared at fixation. The subjects were instructed to decide whether this item was a correctly spelled English word; a "yes" response was made by pressing the " $z$ " key with the left index finger, and a "no" response was made by pressing the "/" key with the right index finger. Response to the target initiated a 750msec intertrial interval, after which the fixation cross appeared, marking the start of a new trial.

Experiment 1. In Experiment 1, the attended prime was a word and the probe character was a letter. There were two groups of subjects; 16 took part in the read condition and 32 in the letter search condition. The subjects in the letter search condition searched the attended prime for the probe letter and made a lexical decision to the target. In the read condition, the subjects were instructed to read the red prime word and make a keypress response when they had done so. The subjects in both the read and letter search conditions were explicitly instructed to ignore the white word (the ignored prime).

Experiment 2. Experiment 2 was identical to the design of the letter search condition of Experiment 1, except that the ignored prime was presented in between the probe string and the attended prime, as is shown in Figure 1.

Experiment 3. Experiment 3 was identical to Experiment 2, except that the attended prime and the probe letter were replaced with an equal number of digits (e.g., a five-letter word was replaced with five digits), as is shown in Figure 1.

\section{Results \\ Overall Data Treatment}

Reaction time. In the read condition of Experiment 1, only data from word target trials where a correct response was made were included in the reaction time (RT) analysis. This resulted in an elimination of $4.7 \%$ of word target trials. The remaining data were then subjected to a recursive outlier analysis where scores falling 3.5 or more standard deviations above or below the mean score (excluding the most extreme score) for that condition were eliminated from further analyses (Van Selst \& Jolicœur, 1994). This resulted in a further elimination of $3.5 \%$ of the trials.

In the letter search condition of Experiments 1 and 2 and in the digit search condition of Experiment 3, only data from word-target trials with correct responses to both the letter/ digit search and lexical decision tasks were included in the analysis.

This resulted in an elimination of $8.4 \%$ of word-target trials in the letter search condition of Experiment 1, 8.2\% in Experiment 2, and $7.9 \%$ in Experiment 3. These data were then subjected to the same outlier procedure described above. This resulted in a further elimination of $3.7 \%, 6.2 \%$, and $5.5 \%$ of the trials for Experiments 1, 2, and 3, respectively. Table 1 presents mean RTs for each prime type for both the read and letter search conditions.

Errors. Table 1 also presents mean percentage error for the attended prime, ignored prime, and unrelated prime conditions for the read, letter search, and digit search conditions. The percentage errors for all conditions, across all experiments, was $5.7 \%$ or less.

\section{Experiment 1}

The effect of ignored prime location was not significant for RTs $(F<1)$ or for errors $(F<1)$ and did not interact with the effect of prime type $\left[F(1,31)=1.1, M S_{\mathrm{e}}=1,312\right.$, $p>.30$ ] or probe type (presence/absence of probe letter in

Table 1

Mean Word RT (in Milliseconds), Standard Deviation (SD), and Error (\% E) as a Function of Experiment, Condition (Read vs. Search), Prime Type (Attended vs. Ignored), and Relatedness (Unrelated vs. Related)

\begin{tabular}{|c|c|c|c|c|c|c|c|c|c|c|c|c|c|}
\hline & \multicolumn{6}{|c|}{ Experiment 1} & \multirow{2}{*}{\multicolumn{3}{|c|}{$\begin{array}{c}\text { Experiment } 2 \\
\text { Letter Search } * *\end{array}$}} & \multirow{2}{*}{\multicolumn{3}{|c|}{$\frac{\text { Experiment } 3}{\text { Digit Search** }}$}} \\
\hline & & \multicolumn{3}{|c|}{ Read* } & \multicolumn{3}{|c|}{ Letter Search** } & & & & & & \\
\hline & & RT & $S D$ & $\% \mathrm{E}$ & RT & $S D$ & $\% \mathrm{E}$ & RT & $S D$ & $\% \mathrm{E}$ & RT & $S D$ & $\% \mathrm{E}$ \\
\hline \multicolumn{2}{|c|}{$\begin{array}{l}\text { Target Processing } \\
\text { (Lexical Decision) }\end{array}$} & & & & & & & & & & & & \\
\hline Prime Type & Relatedness & & & & & & & & & & & & \\
\hline & Related & 624 & 100 & 4.2 & 699 & 129 & 3.4 & 640 & 117 & 3.5 & & & \\
\hline & Difference & 41 & & -0.4 & 0 & & 0.4 & 4 & & 2.1 & & & \\
\hline \multirow[t]{3}{*}{ Ignored } & Unrelated & 665 & 80 & 3.8 & 699 & 118 & 3.8 & 644 & 99 & 5.6 & 642 & 118 & 4.8 \\
\hline & Related & 638 & 97 & 5.0 & 698 & 117 & 2.7 & 642 & 120 & 3.7 & 619 & 112 & 3.6 \\
\hline & Difference & 27 & & -1.2 & 1 & & 1.1 & 2 & & 1.9 & 23 & & 1.2 \\
\hline
\end{tabular}

$* N=16 . \quad * * N=32$. 
attended prime; $F<1$ ). The data were therefore collapsed over this variable in subsequent analyses. The effect of probe type approached significance for RTs $[F(1,31)=$ $\left.2.7, M S_{\mathrm{e}}=1,346, p<.15\right]$ and was significant for errors $\left[F(1,31)=14.1, M S_{\mathrm{e}}=5, p<.01\right]$. However, it did not interact with the effect of prime type for RTs $(F<1)$ or for errors $\left[F(1,31)=1.8, M S_{\mathrm{e}}=8, p>.15\right]$. The main effect of probe type [more errors when the probe letter was present $(4.5 \%)$ in the attended prime than when it was absent $(2.3 \%)]$ may mean that the subjects were biased to indicate that the probe letter was not present when a search did not reveal an immediate match. This result is not central to this study, and, given that probe type did not interact with the effect of prime type or the effect of ignored prime location in terms of RT, the data were collapsed over this variable in subsequent analyses. Given that the critical comparisons here are related versus unrelated for the attended prime and related versus unrelated for the ignored prime, an analysis of these planned comparisons is presented, rather than an omnibus analysis.

Read condition. As can be seen in Table 1, priming was observed in both the attended and the ignored prime conditions. The 41-msec priming effect in the attended prime condition was significant $\left[F(1,15)=10.0, M S_{\mathrm{e}}=1,321\right.$, $p<.01]$. The 27 -msec priming effect in the ignored prime condition was also significant $\left[F(1,15)=6.1, M S_{\mathrm{e}}=950\right.$, $p<.05]$. An analysis of variance (ANOVA) of the error data for the read condition of Experiment 1 did not reveal any main effect of relatedness in either the attended $(F<1)$ or the ignored prime conditions $(F<1.4)$.

Letter search condition. In contrast to the read condition (see Table 1), there was no semantic priming effect in either the attended prime $(F<1)$ or the ignored prime $(F<1)$ conditions. Given that this is a null effect, a power analysis was performed (Cohen, 1988). The letter search condition yielded .98 power to detect a $41-\mathrm{msec}$ priming effect in response to the attended prime and .98 power to detect a $27-\mathrm{msec}$ priming effect in response to the ignored prime. The effect sizes used here are the sizes of the priming effects in the attended and ignored prime conditions of the read condition in Experiment 1, respectively.

An ANOVA of the error data for the letter search condition of Experiment 1 did not reveal any main effect of relatedness in either the attended $(F<1)$ or the ignored prime conditions $\left[F(1,31)=1.7, M S_{\mathrm{e}}=7, p>.15\right]$.

\section{Experiment 2}

The effect of probe type was not significant for either RTs $\left[F(1,31)=1.01, M S_{\mathrm{e}}=339, p>.3\right]$ or errors $(F<1)$ and did not interact with the effect of prime type for either RTs $\left[F(2,62)=1.07, M S_{\mathrm{e}}=1,430, p>.3\right]$ or errors $(F<1)$. The data were thus collapsed over this variable in subsequent analyses. No semantic priming was observed in either the attended prime $(F<1)$ or the ignored prime $\left[F(1,31)=0.029, M S_{\mathrm{e}}=1,828, p>.85\right]$ conditions (see Table 1). Given that this is a null effect, a power analysis was performed. This analysis yielded .99 power to detect a $41-\mathrm{msec}$ priming effect in the attended prime condition and a $27-\mathrm{msec}$ priming effect in the ignored prime condition. The priming effect sizes used here are the sizes of the priming effects in the attended and ignored prime conditions of the read condition in Experiment 1 , respectively.

An ANOVA of the error data for Experiment 2 revealed a main effect of relatedness in both the attended $[F(1,31)=$ $\left.13.75, M S_{\mathrm{e}}=5, p<.005\right]$ and the ignored prime conditions $\left[F(1,31)=8.47, M S_{\mathrm{e}}=7, p<.01\right]$. Clearly, there was a small $(\sim 2.0 \%)$ but significant error effect, though it should be noted that error effects in the letter search paradigm tend to be unstable (Chiappe et al., 1996).

\section{Experiment 3}

The effect of probe type approached significance in the RT data $\left[F(1,31)=3.05, M S_{\mathrm{e}}=354, p<.10\right]$ and was significant in the error data $\left[F(1,31)=10.48, M S_{\mathrm{e}}=4\right.$, $p<.005]$ with faster and more accurate digit search responses for trials in which the probe was present in the attended prime. This result is not surprising given that subjects were most likely searching the attended prime serially and terminated this process when a match was found. More importantly, however, probe type did not interact with the effect of prime type in terms of RT $[F(2,62)=$ $1.48, M S_{\mathrm{e}}=1,185, p>.20$ ] or in terms of error $(F<1)$. The data were thus collapsed over this variable in subsequent analyses. As can be seen in Table 1, a 23-msec priming effect was observed in the ignored prime condition $\left[F(1,31)=4.52, M S_{\mathrm{e}}=1,791, p<.05\right]$. An analysis of the corresponding error data yielded no significant difference $\left[F(1,31)=1.68, M S_{\mathrm{e}}=14, p>.20\right]$.

\section{DISCUSSION}

The results of these three experiments can be summarized as follows. Semantic priming was observed in the read condition of Experiment 1 for both attended and ignored primes. In contrast, there was no semantic priming for either attended or ignored primes in the letter search condition of Experiment 1.3 All subjects performed letter search to a prime in Experiment 2, and the ignored prime appeared at fixation. Semantic priming was not observed for either attended or ignored primes. In Experiment 3, the prime search task was through Arabic numerals, and the ignored (word) prime occurred at fixation. Here, the ignored prime was semantically activated, given that semantic priming was observed.

The observation of priming in the read condition of Experiment 1 is standard (see Neely's, 1991, review). The failure to observe semantic priming for the attended primes following letter search of the prime (Experiments 1 and 2) is also standard (Chiappe et al., 1996; Friedrich, et al., 1991; Henik et al., 1983; Henik et al., 1994; Smith et al., 1983; Stolz \& Besner, 1996, 1998).

The novel finding in Experiment 1 is that semantic priming was not observed for ignored primes in the letter search condition. However, this finding is open to the objection that the processing load associated with the letter search 
task might be higher than in the read condition. If processing were consequently more narrowly focused (spatially), the ignored prime may have suffered because of its peripheral location relative to the attended prime. This possibility was addressed by presenting the ignored prime at fovea in Experiment 2; semantic priming was not observed in the RT data for either attended or ignored primes.

\section{Activation Blocking}

Stolz and Besner's activation block account of the elimination of semantic priming is cast in terms of the IA framework and assumes that information from the lexical level is blocked from reaching the semantic level. One version of this account is that the activation block prevents all information at the lexical level from being processed to the semantic level. Another version is that only the prime item that is being searched is prevented from being processed semantically. The absence of semantic priming in response to both attended and ignored primes in Experiments 1 and 2 in the RT data is consistent with the conclusion that the activation block between lexical and semantic levels is general to all items at the lexical level, rather than being specific to the prime item that is searched for a target letter.

\section{Is Semantic Activation Automatic?}

Neely and Kahan (2001) claim that full-blown semantic activation and therefore full-blown semantic priming should be seen for a foveally presented task-irrelevant word, regardless of the type of processing required on the task-relevant event. The failure to observe semantic priming in RTs in the ignored prime condition of Experiment 2 is inconsistent with this claim. Although there was a priming effect in the error data for both the ignored and attended prime conditions in Experiment 2, this effect was small $(\sim 2.0 \%)$. This is inconsistent with Neely and Kahan's claim that semantic activation for task-irrelevant events should be full blown. ${ }^{4,5}$

\section{Domains of Processing}

Besner and colleagues (Besner \& Stolz, 1999; Besner, Stolz, \& Boutilier, 1997; Chiappe et al., 1996; Smith \& Besner, 2001; Stolz \& Besner, 1996) assume that processing to the semantic level is the default set for the reader. However, when processing is directed to the letter level, an activation block is constructed between lexical and semantic levels of representation. If prime processing involves another domain (e.g., if prime processing refers to a global judgment about color rather than to a decision about an individualletter), an activation block is not set up in the visual word recognition system (Chiappe et al., 1996). Consequently, semantic priming is seen under these circumstances. Experiment 3 of the present series extends this analysis. An ignored prime yields semantic priming in RTs when the prime task requires search through Arabic numerals, ${ }^{6}$ in contrast to the absence of such priming in Experiments 1 and 2 in which the prime task required search through letters.

\section{Conclusions}

The data reported here are inconsistent with contextindependent ${ }^{7}$ automatic semantic activation. In contrast, the data are consistent with an account in which prime search through letters instantiates an activation block between lexical and semantic levels of representation in the visual word recognition system that is general to a level rather than being specific to an item at that level. This activation block is eschewed when prime search is through material from a different domain.

\section{REFERENCES}

Ashby, F. G., \& Townsend, J. T. (1980). Decomposing the reaction time distribution: Pure insertion and selective influence revisited. Journal of Mathematical Psychology, 21, 93-123.

BeSNer, D., \& SMith, M. C. (1992). Models of visual word recognition: When obscuring the stimulus yields a clearer view. Journal of Experimental Psychology: Learning, Memory, \& Cognition, 18, 468-482.

BeSNER, D., \& STOLz, J. A. (1999). Unconsciously contextually controlled processing: The Stroop effect reconsidered. Psychonomic Bulletin \& Review, 6, 449-455.

Besner, D., Stolz, J. A., \& Boutilier, C. (1997). The Stroop effect and the myth of automaticity. Psychonomic Bulletin \& Review, 4, 221-225.

BorowsKy, R., \& BESNER, D. (1991). Visual word recognition across orthographies: On the interaction between context and degradation. Journal of Experimental Psychology: Learning, Memory, \& Cognition, 17, 272-276.

Chiappe, P. R., Smith, M. C., \& Besner, D. (1996). Semantic priming in visual word recognition: Activation blocking and domains of processing. Psychonomic Bulletin \& Review, 3, 249-253.

COHEN, J. (1988). Statistical power analysisfor the behavioral sciences (2nd ed.). Hillsdale, NJ: Erlbaum.

Friedrich, F. J., HeniK, A., \& Tzelgov, J. (1991). Automatic processes in lexical access and spreading activation. Journal of Expermiental Psychology: Human Perception \& Performance, 17, 792-806.

Henderson, L. (1987). Word recognition: A tutorial review. In M. Coltheart (Ed.), Attention and performance XII: The psychology of reading (pp. 171-200). Hillsdale, NJ: Erlbaum.

HeniK, A., Friedrich,F. J., \& Kellogg, W. A. (1983). The dependence of semantic relatedness effects upon prime processing. Memory \& $\mathrm{Cog}$ nition, 11, 366-373.

Henik, A. Friedrich, F. J., Tzelgov, J., \& Tramer, S. (1994). Capacity demands of automatic processes in semantic priming. Memory \& $\mathrm{Cog}$ nition, 22, 157-168.

Johnston, J. C. (1981). Understanding word perception: Clues from studying the word superiority effect. In O. J. L. Tzeng \& H. Singer (Eds.), Perception of print: Reading research in experimental psychology (pp. 65-81). Hillsdale, NJ: Erlbaum.

Logan, G. D., \& Schulkind, M. D. (2000). Parallel memory retrieval in dual-task situations: I. Semantic memory. Journal of Experimental Psychology: Human Perception \& Performance, 26, 1072-1090.

MacLeod, C. (2000, July). Putting automaticity in context: Reducing the Stroop effect. Paper presented at the joint meeting of the Experimental Psychological Society and the Canadian Society for Brain, Behaviour \& Cognitive Science, Cambridge, U.K.

Marí-Beffa, P., Fuentes, L. J., Catena, A., \& Houghton, G. (2000). Semantic priming in the prime task effect: Evidence of automatic semantic processing of distractors. Memory \& Cognition, 28, 635-647.

McClelland, J. L. (1987). The case for interactionism in language processing. In M. Coltheart (Ed.), Attention and performance XII: The psychology of reading (pp. 3-35). Hillsdale, NJ: Erlbaum.

McClelland, J. L., \& Rumelhart, D. E. (1981). An interactive activation model of context effects in letter perception: I. An account of basic findings. Psychological Review, 88, 375-407.

NeELy, J. H. (1991). Semantic priming effects in visual word recognition: A selective review of current findings and theories. In D. Besner \& G. Humphreys (Eds.), Basic processes in reading: Visual word recognition (pp. 264-336). Hillsdale, NJ: Erlbaum. 
Neely, J. H., \& KaHan, T. (2001). Is semantic activation automatic? A critical re-evaluation. In H. L. Roediger, J. S. Nairne, I. Neath, \& A. M. Surprenant (Eds.), The nature of remembering: Essays in honor of Robert G. Crowder (pp. 69-93). Washington, DC: American Psychological Association.

Paap, K. R., Newsome, S. L., MacDonald, J. E., \& Schvaneveldt, R. W. (1982). An activation-verification model for letter and word recognition. Psychological Review, 89, 573-594.

SCHNEIDER, W. (1988). Micro Experimental Laboratory: An integrated system for IBM-PC compatibles. Behavior Research Methods, Instruments, \& Computers, 20, 206-217.

SCHNEIDER, W. (1990). MEL user's guide: Computer techniques for real time experimentation. Pittsburgh, PA: Psychology Software Tools.

Smith, M. C., \& Besner, D. (2001). Modulating semantic feedback in visual word recognition. Psychonomic Bulletin \& Review, 8, 111-117.

Smith, M. C., Besner, D., \& Miyoshi, H. (1994). New limits to automaticity: Context modulated semantic priming. Journal of Experimental Psychology: Learning, Memory, \& Cognition, 20, 104-115.

Smith, M. C., Theodor, L., \& Franklin, P. E. (1983). The relationship between contextual facilitation and depth of processing. Journal of Experimental Psychology: Learning, Memory, \& Cognition, 9, 697-712.

Stolz, J. A., \& Besner, D. (1996). The role of set in visual word recognition: Activation and activation blocking as non-automatic processes. Journal of Experimental Psychology: Human Perception \& Performance, 22, 1166-1177.

Stolz, J. A, \& BeSNER, D. (1998). Levels of representation in visual word recognition: A dissociation between semantic and morphological processing. Journal of Experimental Psychology: Human Perception \& Performance, 24, 1642-1655.

Stolz, J. A., Robidoux, S., \& Besner, D. (2000). Semantic priming in the context of an interactive activation framework: Explorations in connectionist modeling. Manuscript submitted for publication.

TAFT, M. (1991). Reading and the mental lexicon. Hillsdale, NJ: Erlbaum.

VAn Selst, M., \& Jolicceur, P. (1994). A solution to the effect of sample size on outlier elimination. Quarterly Journal of Experimental Psychology, 47A, 631-650.

\section{NOTES}

1. By "eliminated" we mean any remaining difference between related and unrelated conditions is not statistically significant.

2 . The activation block between the lexical and semantic levels is logically independent of the feedback from the lexical level to the letter level. Stolz and Besner $(1996,1998)$ assumed increased feedback from the lexical level to the letter level during letter search because the work is cast in the context of the IA framework. It should be noted, however, that other models of visual word recognition do not assume feedback from the lexical level to the letter level. For example, there are successful accounts of the word superiority effect (and by inference, the letter search task) that do not assume feedback between lexical and letter levels (e.g., Johnston, 1981; Paap, Newsome, MacDonald, \& Schvaneveldt, 1982; see also Smith \& Besner, 2001).

3. Marí-Beffa, Fuentes, Catena, and Houghton (2000) reported that an ignored prime yields semantic priming following letter search on the attended prime, a result that differs from Experiments 1 and 2 of the present series. It should be noted that their procedure differs substantially from the standard preparation in that the letter search probe appeared well before the word to be searched. One consequence is that prime search time was about $500 \mathrm{msec}$ faster than in these and other experiments in the literature. Most centrally, it is unlikely that the inferences Marí-Beffa et al. drew from their paradigm apply to either attended or unattended prime processing in the standard letter search paradigm. In their experiments, substantial semantic priming for attended primes, $[15 \mathrm{msec}$ in Experiment 1 (not significant), and $23 \mathrm{msec}$ in Experiment 2 (significant)] was seen following letter search. These results do not correspond to the standard finding of no semantic priming following letter search on the prime when the letter to be searched for and the prime appear simultaneously.

4. In order to address the possibility of a speed-accuracy tradeoff, Ashby and Townsend's (1980) correction was applied. This correction divides mean RT by the proportion of correct responses for each subject and assumes a linear relationship between speed and accuracy. It is well known that the speed-accuracy function is not linear; however, since the range over which errors occur is so small, one may wish to assume a linear function. This correction failed to produce a priming effect in RTs for either the attended or ignored prime conditions $\left[F(1,31)=2.86, M S_{\mathrm{e}}=\right.$ $2,235, p>.10 ; F(1,31)=1.14, M S_{\mathrm{e}}=3,202, p>.25$, respectively].

5 . We are not willing to accept the conclusion that no semantic priming occurred here, but, at the same time, it can be argued that full-blown semantic priming did not occur.

6 . The phenomenological experience of the digit search prime display is that the ignored prime "pops out."

7. The results reported here are problematic for context independent automatic semantic activation as proposed by Neely and Kahan (2001). Some investigators attempt to salvage the notion of automaticity by making it context dependent (e.g., MacLeod, 2000) or conditional (see Logan $\&$ Schulkind, 2000). The problem with this gambit is that there is nothing in this notion that specifies, a priori, what conditions (context) will and will not enable automatic processing. This line of theorizing seems futile in the absence of such specification.

(Manuscript received July 29, 1999; revision accepted for publication January 10, 2001.)

\section{Forthcoming Articles}

The current list of articles in press for Psychonomic Bulletin \& Review can be found at the Psychonomic Society Publications web site: www.psychonomic.org/PBR/forthcoming.htm 